\title{
1 Numerical determination of vertical water flux based on soil temperature profiles
}

2

3

4

5

6

7

Alain Tabbagh ${ }^{1}$, Bruno Cheviron ${ }^{2}$; Hocine Henine ${ }^{3}$, Roger Guérin ${ }^{1}$, Mohamed-Amine Bechkit ${ }^{4}$

${ }^{1}$ Sorbonne Universités, UPMC Univ Paris 06, UMR 7619, METIS, F-75005, Paris, France

2 Irstea, UMR G-EAU, Domaine de Lavalette, 361 rue Jean-François Breton, 34196 Montpellier cedex 5, France

${ }^{3}$ Irstea, Hydrosystèmes et Bioprocédés, 1 rue Pierre-Gilles de Gennes, CS 10030, 92761 Antony cedex, France

${ }^{4}$ Université des Sciences et de la Technologie Houari Boumediene, BP 32 El Alia, 16111 Bab Ezzouar, Alger, Algeria

Corresponding author: Alain Tabbagh, +33 1 44274824, alain.tabbagh@upmc.fr

Abstract

High sensitivity temperature sensors (0.001 K sensitivity Pt100 thermistors), positioned at intervals of a few centimetres along a vertical soil profile, allow temperature measurements to be made which are sensitive to water flux through the soil. The development of high data storage capabilities now makes it possible to carry out in situ temperature recordings over long periods of time. By directly applying numerical models of convective and conductive heat transfer to experimental data recorded as a function of depth and time, it is possible to calculate Darcy's velocity from the convection transfer term, thus allowing water infiltration/exfiltration through the soil to be determined as a function of time between fixed depths.

In the present study we consider temperature data recorded at the Boissy-le-Châtel (Seine et Marne, France) experimental station between April 16th, 2009 and March 8th, 2010, at six different depths and 10-min time intervals. We make use of two numerical finite element models to solve the conduction/convection heat transfer equation and compare their merits. These two models allow us to calculate the corresponding convective flux rate every 
day using a group of three sensors. The comparison of the two series of calculated values centred at $24 \mathrm{~cm}$ shows reliable results for periods longer than 8 days.

These results are transformed in infiltration/exfiltration value after determining the soil volumetric heat capacity. The comparison with the rainfall and evaporation data for periods of ten days shows a close accordance with the behaviour of the system governed by rainfall evaporation rate during winter and spring.

Keywords: Infiltration, unsaturated soil, numerical finite element models, Pt100 thermistor, temperature

\section{Introduction}

Since convection is a component of the heat transport process, water seepage can be in turn determined through the analysis of temperature measurements [1], [2], [3]. The analysis of the temperature distribution thus offers the possibility to determine the Darcy's velocity without knowing head gradients. Although this is an old idea, it is likely that it will be more extensively developed in the future as a result of the possibilities offered by a large panel of new technologies, which facilitate the acquisition and recording of data. As an example, temperature monitoring with a fibre optic sensor (distributed temperature sensing, DTS) [4], [5], [6], and [7] allows data to be recorded at high temporal and spatial densities and over long distances. High resolution sensors coupled with low power electronics and vast data storage capacities have also contributed to the development of renewed in-field applications involving temperature measurements, surveys and chronicles.

For more than fifty years, soil temperature monitoring has been applied to both saturated and unsaturated underground media. However researchers face a major difficulty: seepage velocities are generally low in temperate climate soil contexts (dominance of clay 
loam, medium water contents, low rainfall intensities) thus the conductive heat transfer largely dominates that due to convection, which makes the Peclet number clearly smaller than 1. Measurements and calculations must therefore be very accurate, and a detailed description of the soil's conductive transfer is required to ensure that the convective component can be correctly evaluated.

The present paper deals with natural heat exchanges which allow the long-term analysis of water seepage. Our approach extends those exposed in a series of prior studies, which can be summarized as follows.

By considering a saturated medium (rice paddies) and high percolation rates, Suzuki [8] was the first to derive a method allowing percolation to be estimated from the amplitude ratio of sinusoidal temperature fluctuations along vertical profiles. Stallman [9] proposed an analytical solution, based on the attenuation of sinusoidal temperature fluctuations, which was applied to the case of an unsaturated medium and diurnal temperature fluctuations, leading to an ultimate accuracy of $1 \mathrm{~mm} \mathrm{~d}^{-1}$. For deeper borehole measurements, where steady state conditions can be assumed, Bredehoeft and Papadopoulos [10] proposed an analytical solution taking both the conductive and convective transfers into account, which leads to an exponential variation of temperature as a function of depth, governed by Darcy's velocity. By making the same assumptions, several authors [11], [12], [13] compared, with satisfactory results, water flows obtained using this approach with those determined from hydrological data. Taniguchi [14] contributed several improvements to the unsteady state analytical approach, taking the amplitude and phase of sinusoidal time variations into account and distinguishing between infiltration and exfiltration. Contrary to Stallman [9], Taniguchi [14] based these calculations on annual temperature fluctuations. Complete analytical solutions to the conductive and convective transfer problem for sinusoidal and transient variations were proposed by Tabbagh et al. [15]. Other studies specifically analysed flow through streambeds 
[16], [17], [18] and attempts have been made to develop a numerical approach for recharge determinations using borehole measurements [19]. Today a significant research effort is still involved in semi-analytical based Fourier models [3], [20]. All of these studies were based on the assumption of a homogeneous medium. Interpretation of layered terrain using analytical solutions is more complex. The study must be split into two steps: firstly determine the thermal structure, and then the Darcy velocity [21]. Such an algorithm has been applied to the determination of recharge rate, in the Seine river basin (France) over a period of several years [22] where a sufficiently dense network of meteorological stations exists. However, the calculation process remains complex. The main limitation of this process is the lack of resolution of the sensors $(0.1 \mathrm{~K}$ in meteorological stations $)$ which must be compensated for by stacking long series of data. However, stacking the data limits the recharge determination to multi-annual cycles, except in the case of significant transient thermal events accompanied by a sufficiently strong thermal signal [23].

In this study, we present a new framework and the first test of direct resolution models that rely on high precision sensors. The sensors are positioned at different depths of several centimetres along a vertical soil profile (see Figure 1) and temperature measurements are collected at short intervals over a period of several months. The infiltration/exfiltration is calculated through the use of simple numerical scheme(s) based on the finite element (FE) method, such that variations in the soil's thermal properties can be determined over a short distance or any desired time interval.

\section{Materials and methods}

\subsection{Instrumentation}

We make use of new Pt100 thermistors (Correge, France, http://www.correge.fr/) with a resolution of $0.001 \mathrm{~K}$, together with a dedicated autonomous acquisition system allowing 
measurement intervals of a few minutes to be achieved with the same $0.001 \mathrm{~K}$ resolution. The sensors and the associate electronics were previously co-calibrated in laboratory in order to correct for the slight offsets that exist between them [24].

The study plot of $614 \mathrm{~m}^{2}$ surface is located at the experimental site of Boissy-le-Châtel in the Orgeval catchment (70 km East of Paris, France) (http://data.datacite.org/10.17180/OBS.ORACLE). The annual average air temperature is $12^{\circ} \mathrm{C}$. The area of this catchment is covered by a quaternary loess deposit whose maximum thickness is $10 \mathrm{~m}$. The top layer has evolved into hydromorphic gleyic luvisol (FAO soil classification) that presents hydromorphic characteristics and may cause the formation of a temporary perched water table in the winter season. The plot is artificially drained by buried perforated pipes (buried at $0.6 \mathrm{~m}$ and separated by about $6 \mathrm{~m}$ ) and managed for experimental purposes, but unfortunately no measurement of the drained quantities was possible in 2009 and 2010. This plot is instrumented for a continuous monitoring (hourly recording) of meteorological variables (air and soil temperature, net radiation, air pressure and relative humidity). Based on the daily average of these variables, "Météo France" calculates potential evapotranspiration by using the Penman formula [26]. This formula is consistent with the canopy of the study site (grass). Rainfall was measured using tipping bucket rain gauge (manufactured by "Précis mécanique", SA) and recorded each hour (the recording device is Danae LC/RTC, from "Alcyr SARL").

For the initial experiment, the temperature sensors were installed at six different depths: $12,15,18,24,32$ and $34 \mathrm{~cm}$ below ground surface, along the wall of an excavated pit (which was later backfilled). The sensors were inserted into horizontally drilled guide-holes (Figure 1), allowing them to be positioned at accurately known depths with inter-sensor intervals ranging between 3 and $12 \mathrm{~cm}$. The thermal diffusivity of the soil is characterised by annual variations, ranging between $0.61 \times 10^{-6} \mathrm{~m}^{2} \mathrm{~s}^{-1}$ during dry periods and $0.43 \times 10^{-6} \mathrm{~m}^{2} \mathrm{~s}^{-1}$ 
during wet periods and can be used to monitor the soil water content [4], [27]. The temperature recorder and electronic equipment were installed in metal boxes placed on the land surface in the lawn. Sensor configuration and data acquisition were achieved via a serial port on a portable micro-computer, using interfaces produced in our laboratory. Continuous recording began on April $16^{\text {th }}, 2009$ and ended on March $8^{\text {th }}, 2010$, corresponding to a total of 327 days, and was interrupted 4 times, on June $30^{\text {th }}$, September $21^{\text {st }}$, December $16^{\text {th }}, 2009$ and February $10^{\text {th }}, 2010$ in order to change the battery. The $12 \mathrm{~cm}$ sensor did not function between May $22^{\text {nd }}$ and June $30^{\text {th }}$, 2009. Data were recorded at 10-min intervals, leading to a total of 144 discrete measurements per 24-hour period. As the calculations are based upon the time evolution of temperature differences between close sensors, a high resolution is required for the temperature monitoring system; this is illustrated in Figure 2, which plots the variations of the temperature recorded on April 16th and 17th, 2009, and of the difference between two sensors. The temperature variation shows both an amplitude decrease and a phase lag increase with depth. For each curve are drawn the data directly recorded with a $0.001 \mathrm{~K}$ resolution and the data which we would have with a $0.1 \mathrm{~K}$ resolution. Figures $2 \mathrm{~b}$ and $2 \mathrm{c}$ show the difference of temperature between couples of sensors, they demonstrate the significant differences between the $0.1 \mathrm{~K}$ and $0.001 \mathrm{~K}$ resolutions.

\subsection{Calculations}

We assume that the heat generated/absorbed by vaporization, condensation, chemical or biologic activity can be neglected in the considered range of depth, so as the mass and thermal fluxes associated with vapour diffusion. Consequently in absence of local heat source or sink the unsteady conductive heat transfer is governed by the thermal diffusivity, $\Gamma$, and the unsteady convective heat transfer by the flux rate, $v$, and the temperature distribution is thus 
controlled by these two parameters only. When only considering the vertical dimension, $\mathrm{z}$ (1D geometrical problem), the heat equation is expressed as:

$$
\frac{\partial}{\partial z}\left(\Gamma \frac{\partial T}{\partial z}\right)-\frac{\partial}{\partial z}(v T)-\frac{\partial T}{\partial t}=0
$$

The diffusivity $\left(\mathrm{m}^{2} \mathrm{~s}^{-1}\right)$ of the three-phase soil integrates both the thermal conductivity, $\lambda$ $\left(\mathrm{W} \mathrm{m} \mathrm{m}^{-1} \mathrm{~K}^{-1}\right)$ and the volumetric heat capacity, $C_{v}$ in $\left(\mathrm{J} \mathrm{m}^{-3} \mathrm{~K}^{-1}\right)$, whereas the flow rate $\left(\mathrm{m} \mathrm{s}^{-1}\right)$ integrates the Darcy's velocity, $u$, and the ratio of the volumetric capacity of the fluid, $\mathrm{C}_{\mathrm{w}}$, to that of the three-phase medium:

$$
\Gamma(z, t)=\frac{\lambda(z, t)}{C_{v}(z, t)}
$$

$$
v(z, t)=\frac{u(z, t) C_{w}}{C_{v}(z, t)}
$$

When using the FE method equation (1) is integrated over definite size elements. To achieve this integration the variations of all parameters must be chosen. By applying the Galerkin method to triangular two-dimensional (2D) elements defined in the dimensions of depth and time, it is possible to start from this second order differential equation and to integrate by parts using linear variations on the elements.

As illustrated by Figure 1, only three different depths are needed, corresponding to the spatial limits defined by the elements [i-1,i] and [i, i+1] of respective steps $h_{\mathrm{i}}$ and $h_{i+1}$. The time variable lies within the two steps: $[\mathrm{m}-1, \mathrm{~m}]$ and $[\mathrm{m}, \mathrm{m}+1]$ of constant size $\tau . \Gamma$ and $v$ are defined at three consecutive spatial nodes and assumed to vary linearly in $z$ over each element. Because, following equation (1), only the temperature exhibits time derivation, there is no possibility at a given time step to consider a variation of $\Gamma$ and $v$ with time, they are thus constant but they vary with considered time intervals. Thus one uses six spatial unknowns $\Gamma_{i-}$ 1, $\Gamma_{i}, \Gamma_{i+1}, v_{i-1}, v_{i}$ and $v_{i+1}$. Depending on the number of nodes considered in the spatial and time discretization, two models are proposed (Figure 1): the first model involves nine nodes 
and the second use five nodes by omitting the corner nodes. The discretization of equation (1)

175 with the nine-node model yields:

176

$$
\begin{aligned}
& \frac{\tau}{2 h_{i+1}}\left(\Gamma_{i}+\Gamma_{i+1}\right)\left[\frac{2}{3}\left(T_{i+1}^{m}-T_{i}^{m}\right)+\frac{1}{6}\left(T_{i+1}^{m+1}-T_{i}^{m+1}+T_{i+1}^{m-1}-T_{i}^{m-1}\right)\right] \\
& -\frac{\tau}{2 h_{i}}\left(\Gamma_{i}+\Gamma_{i-1}\right)\left[\frac{2}{3}\left(T_{i}^{m}-T_{i-1}^{m}\right)+\frac{1}{6}\left(T_{i}^{m+1}-T_{i-1}^{m+1}+T_{i}^{m-1}-T_{i-1}^{m-1}\right)\right] \\
& -\frac{v_{i} \tau}{9}\left[4 T_{i}^{m}+T_{i-1}^{m}+T_{i+1}^{m}+T_{i}^{m-1}+T_{i}^{m+1}+\frac{1}{4}\left(T_{i-1}^{m-1}+T_{i-1}^{m+1}+T_{i+1}^{m-1}+T_{i+1}^{m+1}\right)\right] \\
& -\frac{v_{i-1} \tau}{9}\left[2 T_{i-1}^{m}+T_{i}^{m}+\frac{1}{2}\left(T_{i-1}^{m-1}+T_{i-1}^{m+1}\right)+\frac{1}{4}\left(T_{i}^{m-1}+T_{i}^{m+1}\right)\right] \\
& -\frac{v_{i+1} \tau}{9}\left[2 T_{i+1}^{m}+T_{i}^{m}+\frac{1}{2}\left(T_{i+1}^{m-1}+T_{i+1}^{m+1}\right)+\frac{1}{4}\left(T_{i}^{m-1}+T_{i}^{m+1}\right)\right] \\
& =\frac{1}{2}\left[\left(T_{i}^{m+1}-T_{i}^{m-1}\right)\left(\frac{h_{i+1}+h_{i}}{3}\right)+\left(T_{i-1}^{m+1}-T_{i-1}^{m-1}\right) \frac{h_{i}}{6}+\left(T_{i+1}^{m+1}-T_{i+1}^{m-1}\right) \frac{h_{i+1}}{6}\right]
\end{aligned}
$$

177 The discretization of equation (1) with the five-node model yields a shorter expression:

$$
-\frac{\tau}{2 h_{i}}\left(\Gamma_{i}+\Gamma_{i-1}\right)\left(T_{i}^{m}-T_{i-1}^{m}\right)+\frac{\tau}{2 h_{i+1}}\left(\Gamma_{i}+\Gamma_{i+1}\right)\left(T_{i+1}^{m}-T_{i}^{m}\right)
$$

$-\frac{v_{i} \tau}{6}\left(4 T_{i}^{m}+T_{i-1}^{m}+T_{i+1}^{m}\right)-\frac{v_{i-1} \tau}{6}\left(2 T_{i-1}^{m}+T_{i}^{m}\right)-\frac{v_{i+1} \tau}{6}\left(2 T_{i+1}^{m}+T_{i}^{m}\right)$

$=\frac{h_{i+1}+h_{i}}{4}\left(T_{i}^{m+1}-T_{i}^{m-1}\right)$

179 The linear expressions (4) and (5) allow the diffusivity and convection terms to be calculated 180 directly from known values of temperature, depth of the sensors and sampling time steps.

Moreover, the use of a 10-min time step makes it possible to verify the stability condition:

$182 \quad \frac{\Gamma \tau}{h^{2}} \leq \frac{1}{2}$

over a wide range of diffusivities (this corresponds to $\Gamma<0.75 \times 10^{-6} \mathrm{~m}^{2} \mathrm{~s}^{-1}$ for $h=3 \mathrm{~cm}$ and $\Gamma<3 \times 10^{-6} \mathrm{~m}^{2} \mathrm{~s}^{-1}$ for $h=6 \mathrm{~cm}$ ) allowing most situations encountered in the field to be covered. to determine the values of $\Gamma$ and $v$, so as to perform crosschecking and evaluate their 
robustness. Following a series of tests both on synthetic data generated by analytical calculation (using realistic soil properties and temperature variations) and Boissy-le-Châtel data, the more stable solution was to consider successive temperatures at levels $i-1$ and $i+1$ as Dirichlet limiting conditions, then to search for the values of $\Gamma_{i-1}, \Gamma_{i}, \Gamma_{i+1}, v_{i-1}, v_{i}$ and $v_{i+1}$ allowing the best (least squares) fit between the calculated and recorded values of $T_{i, m}$ over a sufficiently long calculation interval. The later was taken as the diurnal cycle (i.e. 144 time steps of $10 \mathrm{~min}$ ) or a multiple of it. The computational workflow can thus be broken down into two steps:

- definition of the a priori values: $u=0$ and $\Gamma_{i-1}=\Gamma_{i}=\Gamma_{i+1}$, the latter of which being equal to the optimal least squares value computed using finite differences applied to the simple conduction equation,

- application of a damped least squares process [28] to calculate the six unknowns in equations (4) or (5) where the convergence of the process is controlled by the minimum of the criterion $\mathrm{S}$ defined by:

$$
S=\sqrt{\left(\frac{\delta \Gamma_{i-1}}{\Gamma_{i-1}}\right)^{2}+\left(\frac{\delta \Gamma_{i}}{\Gamma_{i}}\right)^{2}+\left(\frac{\delta \Gamma_{i+1}}{\Gamma_{i+1}}\right)^{2}}+\mu\left|\delta v_{i-1}+2 \delta v_{i}+\delta v_{i+1}\right|
$$

$$
\text { with } \mu=10^{7} \text { if } \nu \text { is expressed in } \mathrm{m} \mathrm{s}^{-1} \text {. }
$$

This process allows taking into account the significant difference in magnitude between the conductive and convective heat fluxes. As an example, for a $1.5 \mathrm{~W} \mathrm{~m}^{-1} \mathrm{~K}^{-1}$ conductivity and a temperature difference of $1 \mathrm{~K}$ over $10 \mathrm{~cm}$ (see Figure 1) the order of magnitude of the conductive heat flux is $15 \mathrm{~W} \mathrm{~m}^{-2}$. For a $4 \mathrm{~mm} \mathrm{~d}^{-1}$ Darcy velocity and a fluid temperature differing of $1 \mathrm{~K}$ from the reference temperature, the order of magnitude of the convective heat 
flux is $0.14 \mathrm{~W} \mathrm{~m}^{-2}$. However the limited range of variation of the thermal diffusivity stabilizes the numerical results.

\section{Results of the calculation and discussion}

The choice of high precision sensors prevents uncertainties resulting from temperature measurements but the choice of simple numeric schemes to describe the time and depth variations of the temperature may be too crude to deliver accurate values of $\Gamma$ and $v$. To assess this issue we compare at a given central depth, $z=24 \mathrm{~cm}$, the two numerical schemes (equations (4) and (5)) with a triad of sensors located at 15, 24 and $34 \mathrm{~cm}$. Figure 3 plots the results of the calculations of $v_{i}$ centred at $24 \mathrm{~cm}$ showing the calculated daily values (thin line) and 10 days values (thick line) using five node-equation (4) (in blue) and nine node-equation (5) (in red). Globally the 10 days values exhibit very coherent results in accordance with the general vegetation behaviour: the calculated flow is upward during spring and the beginning of summer, followed by a downward flow during autumn and winter. On the other hand daily values exhibit a significant level of noise which forbids their direct use. The same difference between daily and 10-day calculations arises for the determination of diffusivity (Figure 4).

The differences between the two numerical schemes stay very small here, except at one point at the end of June where data are missing.

The role of the considered time interval in the calculations results from two facts: (1) the geometric scale of the temperature sensor locations, (2) the linear depth and time variations adopted in the F.E. schemes. The choice of the geometric scale derives from both the respect of the 'Elementary Representative Volume' (at least centimetric) and of the diameter of the sensor encapsulation $(5.7 \mathrm{~mm})$. In the context of this study, because of small

Darcy velocities, the transit time between two sensors necessitates several days (with a 3 mm. $\mathrm{d}^{-1}$ velocity a $3 \mathrm{~cm}$ distance is travelled in 10 days). The differences that may result from 
the imperfect fit between linear schemes and the actual time and depth variations is illustrated by the discrepancies between the results obtained by the 5-node and the 9-node schemes. However, these discrepancies remain lower than $1 \mathrm{~mm} \mathrm{~d}^{-1}$ in the 10 days calculations presented here and the 9-node scheme predicts slightly greater amplitudes.

To assess the robustness of calculations one first considers the mean quadratic deviations when the vertical location of one of the sensors is moved by $1 \mathrm{~mm}$ for a one diurnal cycle interval calculation (Table 1). As could be expected, the deviations are maximal when the central sensor is moved (introducing variations in two pairs of depths instead of one) but they remain limited to a far less than $1 \mathrm{~mm} \mathrm{~d}^{-1}$.

The elementary statistics for the two one diurnal cycle calculations are presented in Table 2. They show an absence of bias, all the means and medians remaining in a $0.4 \mathrm{~mm} \mathrm{~d}^{-1}$ interval, and a greater variability in the nine-point scheme results than in the five-point one. The coherences (the correlation coefficient between two spectra) between the curves are very high (Table 3) but the partition of each spectrum in four quarters (for a 1 day time step, the spectrum extends from 0 to $0.5 \mathrm{~d}^{-1}$ frequency and this interval is divided in four parts) shows that the coherence originates in the first quarter, that is for frequencies lower than $0.125 \mathrm{~d}^{-1}$ (periods of 8 days). The coherences obtained when comparing the flow rates calculated with equation (5) for two different groups of sensors [15, 24 and $34 \mathrm{~cm}]$ and [18, 24 and $32 \mathrm{~cm}$ ] (Table 4) also exhibits a very high value for the first quarter. These strong coherences therefore demonstrate that the water movement is reliably determined for slow temporal variations.

\section{Determination of the Darcy velocity}

For the following steps of the infiltration/exfiltration calculation we will thus use the 'best' of the available results: those having the lowest variance, i.e. the lowest interquartile 


$$
C_{v}=\frac{\alpha}{\Gamma-\beta}
$$

279 where $\alpha=(0.8908-1.0959 n) \lambda_{s}-\frac{C_{s}}{C_{w}}(1-n)(1.2236-0.3485 n)$

$$
\lambda=(0.8908-1.0959 n) \lambda_{s}+(1.2236-0.3485 n) \theta
$$
conductivity).

$$
\text { (10), }
$$

where $C_{s}$ is the volumetric heat capacity of the solid fraction, $n$ the porosity and $\theta$ the volumetric water content. The second relationship, obtained by combining empirical data and numerical modelling, was proposed for the thermal conductivity by Cosenza et al. [30]:

where $\lambda_{s}$ is the thermal conductivity of the solid fraction (also noting that the two first numerical constant are dimensionless while the two others have the dimension of a thermal

In these two relationships $C_{w}$ is constant $\left(C_{w}=4.185 \mathrm{MJ} \mathrm{m}^{-3} \mathrm{~K}^{-1}\right), C_{s}$ can be considered as constant $\left(C_{s}=2.0 \mathrm{MJ} \mathrm{m}^{-3} \mathrm{~K}^{-1}\right)$, while $\lambda_{s}$ and $n$ are variable with $z$ (and site dependent) but constant with $t$; only $\theta$ is time variable. Both (8) and (9) have a linear dependence on $\theta$. Consequently, their combination allows eliminating $\theta$ which results in a direct correspondence between the volumetric heat capacity $\left(C_{v}\right)$ and the thermal diffusivity $(\Gamma)$ : 
281 For the case of the Boissy-le-Châtel site at $24 \mathrm{~cm}$ depth one has $n=0.48$ and $\lambda_{s}=2.15 \mathrm{~W} \mathrm{~m}^{-1} \mathrm{~K}^{-}$ ${ }^{1}, \alpha=0.5218 \mathrm{~W} \mathrm{~m}^{-1} \mathrm{~K}^{-1}$ and $\beta=0.2523 \times 10^{-6} \mathrm{~m}^{2} \mathrm{~s}^{-1}$.

The simulated Darcy velocities, calculated over ten-day periods, were compared with the surface rainfall and Penman potential evapotranspiration (PET) at Boissy-le-Châtel (Figure 5). In an overview, this figure shows negative infiltration rates during spring and the beginning of summer (from April $16^{\text {th }}$ to middle July) then positive infiltration rates with higher values during winter (from December $16^{\text {th }}, 2009$ to March $8^{\text {th }}, 2010$ ). In the first period upwards water movements dominate, they likely result from capillarity and hydraulic gradients created by root water uptake in the first $10 \mathrm{~cm}$ above. The calculated negative infiltration decreases and crosses zero value in July. In July and August, the model results show a positive infiltration while no rainfall occurs and potential evapotranspiration is high, but with a probable low real evapotranspiration (mainly due to the evaporation part as the roots were mostly inactive). In October and the beginning of November the infiltration is small at $24 \mathrm{~cm}$ while the rain is high and the potential evapotranspiration small. In accordance with these two observations, the correlation function between rain at soil surface and infiltration at $24 \mathrm{~cm}$ shows a maximum for a 75 day delay on the total period of 327 days. In winter period nearly saturated soils favour downwards water movements that follow gravity. the most superficial layers tend to hamper water displacements.

For the whole period, the calculated infiltration is negatively correlated with PET (the Pearson coefficient is -0.61 for the 327 day period). The global recharge measured over this period was $158 \mathrm{~mm}$. 
Whereas the present paper establishes the feasibility of the direct calculation of water movements from triads of high-resolution temperature sensors, it seems daring to draw general conclusions about the robustness, applicability and limits of the method: other experiments in similar and different soil contexts are certainly necessary. These will start after delineating the requirements about the measurement parameters themselves, first with indications on the spatial and temporal patterns of data acquisition, then with the most crucial argument on the resolution of the temperature measurements.

- The choice of the vertical spacing (section 3 above) is limited by the soil inhomogeneity (REV) and by the size of the sensor encapsulation. confirmed by looking at the variograms (Figures $6 \mathrm{~b}$ and $7 \mathrm{~b}$ ): at $0.001 \mathrm{~K}$ resolution, the variogram level remains smaller than at $0.01 \mathrm{~K}$ or $0.1 \mathrm{~K}$ with a reduced nugget effect, and a three variograms remaining small. This is explained by the dominance of the conduction transfer over the convection one, for which the $0.001 \mathrm{~K}$ resolution is required in this experiment. 
In the soil and climate conditions considered here, the heat transfer by conduction is usually about one order of magnitude larger than the convection transfer in the unsaturated soil, which makes difficult the determination of the Darcy velocity from temperature measurements. However, the direct calculation of this velocity is of high interest and possible with temperature sensors of sufficient resolution, for time periods greater than a week, with minimal assumptions about soil structure and characteristics. Moreover, the method neither requires the prior knowledge of the hydrodynamic parameters nor any assumption regarding the form of the temperature variations with time and depth.

In summary, we have presented here the first experiment where the limitations resulting from the use of conventional low-sensitivity $(0.1 \mathrm{~K})$ temperature sensors are overcome by using $0.001 \mathrm{~K}$ sensors. Rather than complex analytical calculations we adopted simple FE numerical schemes and a (least squares) stack over multiples of the $24 \mathrm{~h}$ period. The recording of temperature measurements at centimetric spatial and several minutes temporal intervals, and the use of simple numerical models are straightforward and relatively uncomplicated when compared to other more common techniques, such as lysimeters, used for the in situ determination of infiltration and recharge. The implementation of the whole system (sensors, computational tools) stays rather cheap.

The daily values calculated with two different numerical schemes yet exhibits a significant dispersion but this dispersion corresponds to higher frequencies and the coherence of the lower frequency variations are very high; the ten-day periods results are reliable in accordance with local potential flux data. To go further and to reach a day to day determination of the infiltration would necessitate reducing the distance between the sensors due to the order of magnitude of the Darcy's velocity: a few millimetres per day. This 
corresponds to a great challenge because too small geometric scales can be incompatible with

356 the representation of the soil by a continuous medium. Conversely, contexts characterised by

357 higher seepage velocities would be more favourable for the method.

The methodological development exposed here should be considered as a new tool in an 359 expanding toolbox, which can allow new avenues to be explored in the study of critical zone 360 water displacements, in both hydrological and agricultural fields of application. Future progresses would especially address $2 \mathrm{D}$ and $3 \mathrm{D}$ problems or the inclusion of additional terms

362 in the heat equation to handle thermal fluxes in the vapour phase [31], a possible objective 363 being the location of the evaporation front. High precision temperature measurements also 364 merit to be tested for distinguishing between the different types of liquid water flows in soils, 365 typically in the micro- and macro-porosity [32]. 


\section{Acknowledgments}

368

The present study would not have been possible without the support of the IRSTEA

369 (French Research Institute for Science and Technology in the Environment and Agriculture,

370 previously the Cemagref), which provided the authors with access to its BD_ORACLE

371 database (http://data.datacite.org/10.17180/OBS.ORACLE) and relevant complementary data,

372 as well as to the experimental site at Boissy-le-Châtel (Seine et Marne, France) part of the

373 critical zone observatory ORACLE.

374 


\section{References}

[1] Anderson M. P., Heat as a ground water tracer. Groundwater 2005; 43: 951-968.

[2] Constantz J., Heat as a tracer to determine streambed water exchanges. Water Resources Research 2008; 44: W00D10 doi:10.1029/2008WR00699.

[3] Rau G. C., Andersen M. S., McCallum A. M. Roshan H. and Acworth R. I., Heat as a tracer to quantify water flow in near-surface sediments. Earth-Science Review 2014; 129: 4058.

[4] Steele-Dunne S., Rutten M., Krzeminska D., Hausner M., Tyler S. W., Selker J.,Bogaard T. and van De Giesen N., Feasibility of soil moisture estimation using passive distributed temperature sensing. Water Resources Research 2010, 46-3, W03534.

[5] Slater L. D., Ntarlagiannis D., Day-Lewis F. D., Mwakanyamale K., Versteeg R. J., Ward A., Strickland C., Johnson C. D. and Lane Jr. J. W., Use of electrical imaging and distributed temperature sensing methods to characterize surface water groundwater exchange regulating uranium transport at the Hanford 300 Area, Washington. Water Resources Research 2010; 46: W10533 doi:10:1029/2010WR009110.

[6] Briggs M. A., Lautz L. K., McKenzie J. M., Gordon R. P. and Hare D. K., Using highresolution distributed temperature sensing to quantify spatial and temporal variability in vertical hyporheic flux. Water Resources Research 2012; 48: W02527.

[7] Read T., Bour O., Bense V., Le Borgne T., Goderniaux P., Klepikova M.V., Hochreutener R., Lavenant N. and Boschero V., Characterizing groundwater flow and heat transport in fractured rock using fiber-optic distributed temperature sensing. Geophysical Research Letters 2013; 40: 2055-2059.

[8] Suzuki S., Percolation measurements based on heat flow through soil with special reference to paddy fields. Journal of Geophysical Research 1960; 65: 2883-2885. 
[9] Stallman R. W., Steady one-dimensional fluid flow in a semi-infinite porous medium with sinusoidal surface temperature. Journal of Geophysical Research 1965; 70: 2821-2827.

[10] Bredehoeft J. D. and Papadopoulos I., Rates of vertical groundwater movement estimated form the Earth's thermal profiles. Water Resources Research 1965; 1: 325-328.

[11] Stallman R. W., Flow in the zone of aeration. Advances in Hydroscience. Academic Press, New York, 1967; 4: 151-195.

[12] Sorey M. L., Measurement of vertical groundwater velocity from temperature profiles in wells. Water Resources Research 1971; 7: 963-970.

[13] Boyle J. M. and Saleem Z. A., Determination of recharge rates using temperature-depth profiles in wells. Water Resources Research 1979; 15: 1616-1622.

[14] Taniguchi M., Evaluation of vertical groundwater fluxes and thermal properties of aquifers based on transient temperature-depth profiles. Water Resources Research 1993; 29: 2021-2026.

[15] Tabbagh A., Bendjoudi H. and Benderitter Y., Determination of recharge in unsaturated soils using temperature monitoring. Water Resources Research 1999; 35: 2439-2446.

[16] Constantz J., Stewart A. E., Niswonger R., and Sarma L., Analysis of temperature profile for investigating stream losses beneath ephemeral channels, Water Resources Research 2002; 38:1316 doi:10.1029/2001WR001221.

[17] Keery J., Binley A., Crook N. and Smith J. W. N., Temporal and spatial variability of groundwater-surface water fluxes: Development and application of an analytical method using temperature time series. Journal of Hydrology 2007; 336: 1-16.

[18] Roshan H., Rau G. C., Andersen M. S. and Acworth I. R., Use of heat as tracer to quantify vertical streambed flow in a two-dimensional flow field. Water Resources Research 2012; 48: W10508 doi:10.1029/2012WR011918. 
[19] Keshari A. K. and Koo M.-H., A numerical model for estimating groundwater flux from subsurface temperature profiles. Hydrological Processes 2007; 21: 3440-3448.

[20] Halloran L. J. S., Roshan H., Rau G. C., Andersen M. S., Calculating water saturation from passive temperature measurements in near-surface sediments: Development of a semianalytical model, Advances in Water resources2016; 89: 67-79.

[21] Cheviron B., Guérin R., Tabbagh A. and Bendjoudi H., Determining long-term effective groundwater recharge by analysing vertical soil temperature profiles at meteorological stations. Water Resources Research 2005; 41: W09501, doi:10.1029/2005WR004174.

[22] Tabbagh A., Guérin R., Bendjoudi H., Cheviron B. and Bechkit M.-A., Pluri-annual recharge assessment using vertical soil temperature profiles: Example of the Seine river basin (1984-2001). Comptes Rendus Geoscience 2009; 341: 949-956.

[23] Bendjoudi H., Cheviron B., Guérin R. and Tabbagh A., Determination of upward/downward groundwater fluxes using transient variations of soil profile temperature: test of the method with Voyons (Aube, France) experimental data. Hydrological Processes $2005 ; 19: 3735-3745$.

[24] Bechkit M.-A., Sur la détermination de la teneur en eau et de l'infiltration à partir de mesures passives de la température du sol. Thèse de doctorat, Université Pierre et Marie Curie-Paris $62011 ; 162$ p.

[25] Mouhri A., Flipo N., Rejiba F., de Fouquet C., Bodet L., Kurtulus B., Tallec G., Durand V., Jost A., Ansart P. and Goblet P., Designing a multi-scale sampling system of streamaquifer interfaces in a sedimentary basin. Journal of Hydrology 2013; 504: 194-206.

[26] Penman H. L., Natural evaporation from open water, bare soil and grass. Proceedings of the Royal Society London 1948; A193: 120-146.

[27] Bechkit M.-A., Flageul S., Guérin R. and Tabbagh A., Monitoring soil water content by vertical temperature variations. Groundwater 2014; 52: 566-572. 
448 [28] Marquardt D. W., An algorithm for least-square estimation of nonlinear parameters.

449 Journal of the Society for Industrial and Applied Mathematics 1963; 11: 431-441.

450 [29] de Vries D.A., Thermal properties of soils. In: Physics of Plant Environment, edited by

451 W.R. van Wijk, North-Holland Publishing Company, Amsterdam 1963; 210-235.

452 [30] Cosenza P., Guérin R. and Tabbagh A., Relationship between thermal conductivity and 453 water content of soils using numerical modelling. European Journal of Soil Science 2003; 54:

$454 \quad 581-587$.

455 [31] Saito H., Simunek J., Mohanty B. P. Numerical analysis of coupled water, vapour and 456 heat transport in the vadose zone. Vadose Zone Journal 2006; 5: 784-800.

457 [32] Vogel T., Dohnal M., Votrubova J., Modelling heat fluxes in macroporous soil under 458 sparse young forest of temperate humid climate. Journal of Hydrology 2011; 402: 367-376. 
Figure 1: Sensor installation at Boissy-le-Châtel experimental station: location, horizontal holes for the insertion in the wall of the excavated pit, FE depth and time scheme.

Figure 2: Plot of the temperatures (a) and of the differences (b) in temperature variations at 10 min intervals recorded with a $0.001 \mathrm{~K}$ sensitivity on April $16^{\text {th }}$ and $17^{\text {th }}, 2009$ at the Boissy-le-Châtel experimental station. For comparison to existing technology, the recorded data we would have at each sensor for a 0.1 resolution is shown and the plot (c) details the grey zone of plot (b).

Figure 3: Convective flux rate at $24 \mathrm{~cm}$ determined by the two different calculation schemes (equation (4) in red and equation (5) in blue). The daily values correspond to thin lines and the 10 days values to thick lines.

473

Figure 4: Thermal diffusivity at $24 \mathrm{~cm}$ determined by the two different calculation schemes (equation (4) in red and equation (5) in blue). The daily values correspond to thin lines and the 10 days values to thick lines.

477

Figure 5: Comparison between infiltration values calculated for 10 days intervals, surface rainfall and potential evapotranspiration (rain, in red, PET in blue).

Figure 6: (a) Time variations of the flow rates obtained for one day periods with the $0.001 \mathrm{~K}$ resolution (red line), $0.01 \mathrm{~K}$ resolution (green line) and $0.1 \mathrm{~K}$ resolution (blue line), (b) variogram of the flow rate calculated with one day periods, (c) variogram of the thermal diffusivity calculated with one day periods. 
486 Figure 7: (a) Time variations of the flow rates obtained for ten days periods with the $0.001 \mathrm{~K}$

487 resolution (red line), $0.01 \mathrm{~K}$ resolution (green line) and $0.1 \mathrm{~K}$ resolution (blue line), (b)

488 variogram of the flow rate calculated with ten days periods, (c) variogram of the thermal 489 diffusivity calculated with ten days periods.

490 
492 Table captions

493 Table 1: Mean quadratic deviations, $e=\frac{1}{N} \sum_{1}^{N} \sqrt{\left(v_{0, i}-v_{1, i}\right)^{2}}$, between the calculated flow 494 rate with exact sensor location, $v_{0}$, and the flow rate when one sensor is moved of $1 \mathrm{~mm}, v_{l}$.

495

496 Table 2: Means, standard deviations, medians and interquartile distances delivered by the two 497 different calculation schemes.

498

499 Table 3: Coherences for the different parts of the spectrum between the two different 500 calculation schemes.

501

502 Table 4: Coherences for the different parts of the spectrum between the two different triads of 503 sensors $(15,24$ and $34 \mathrm{~cm})$ and $(18,24$ and $32 \mathrm{~cm})$. 


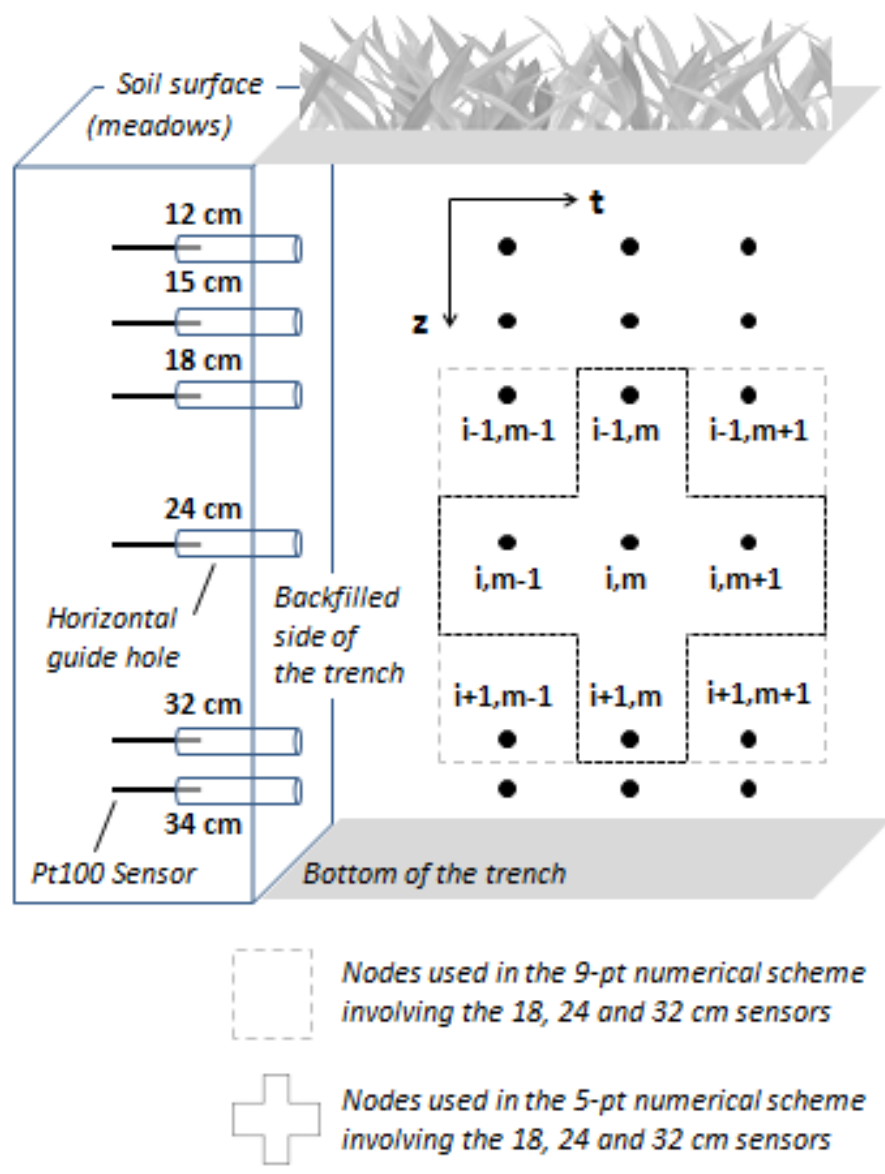

505

506

507 Fig. 1 

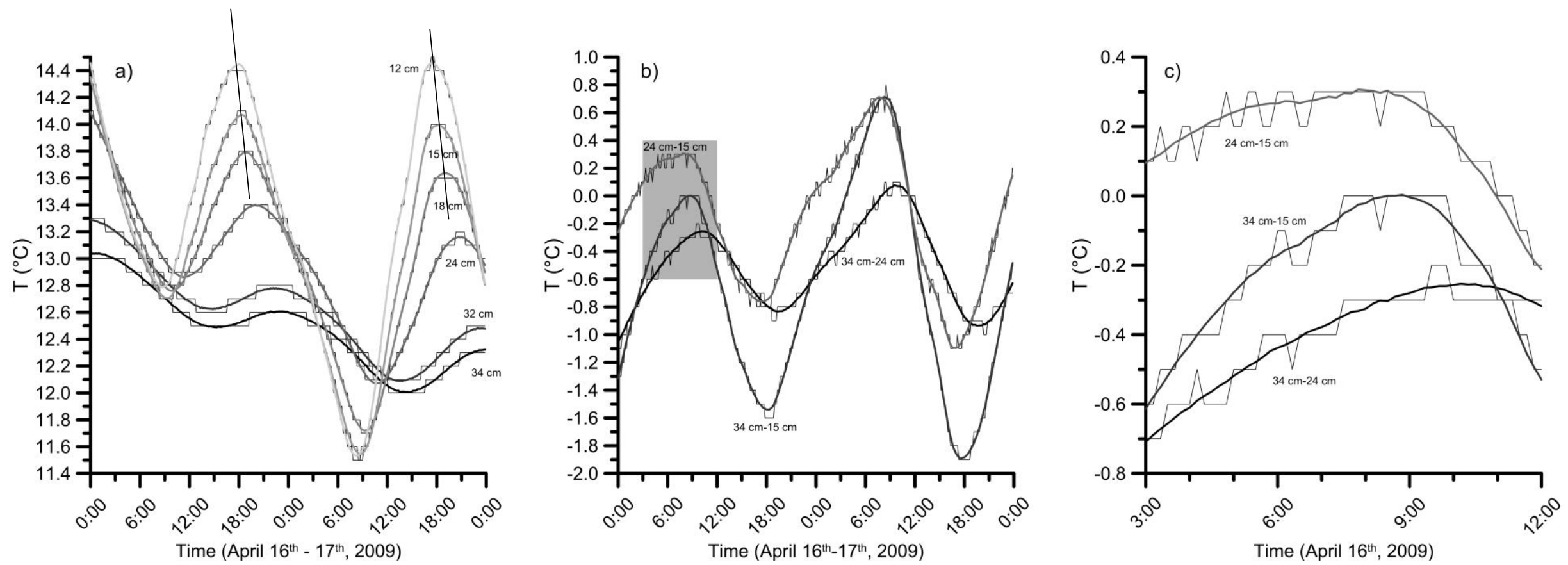

509 Fig. 2 


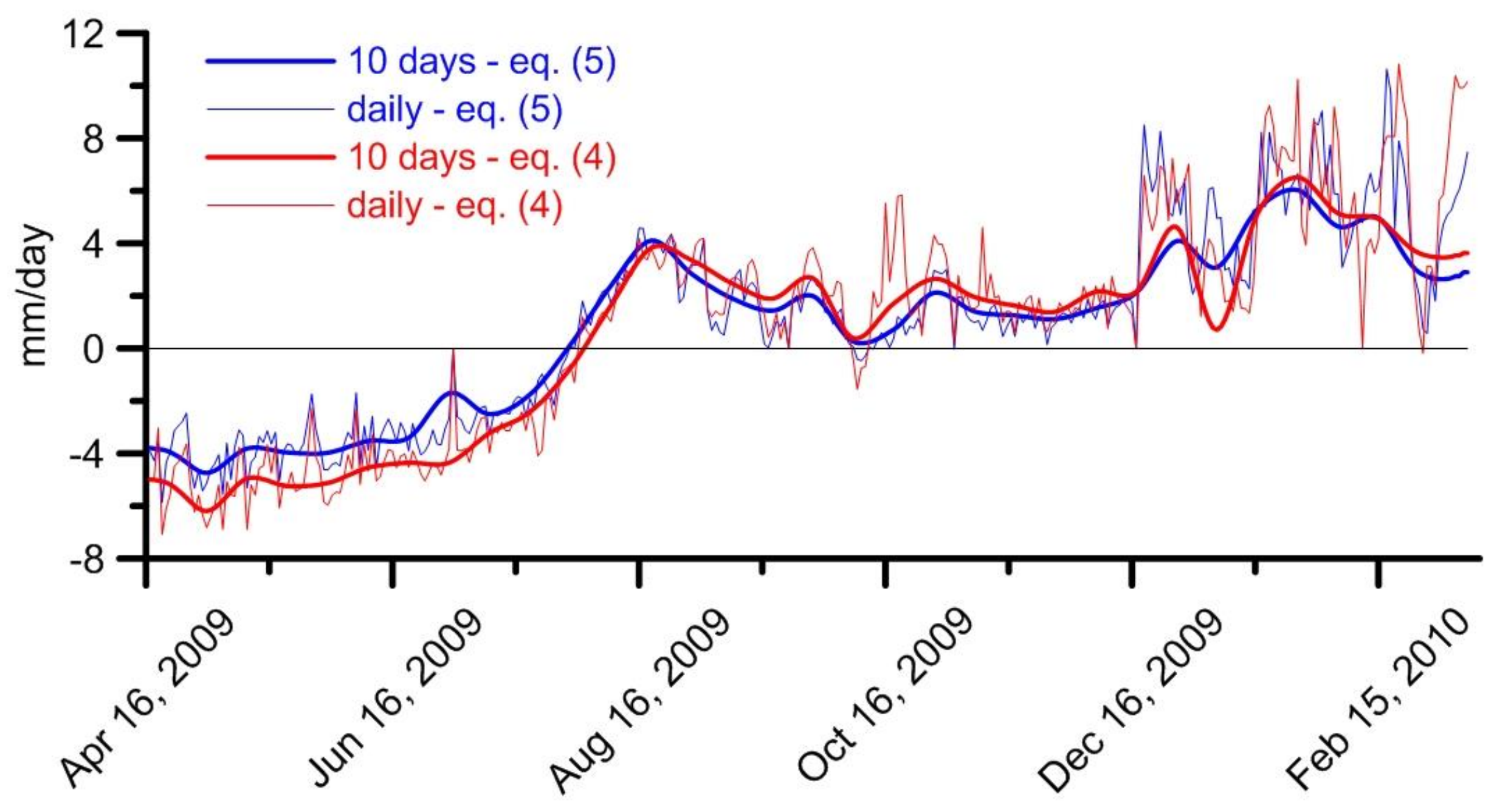

$513 \quad$ Fig. 3 


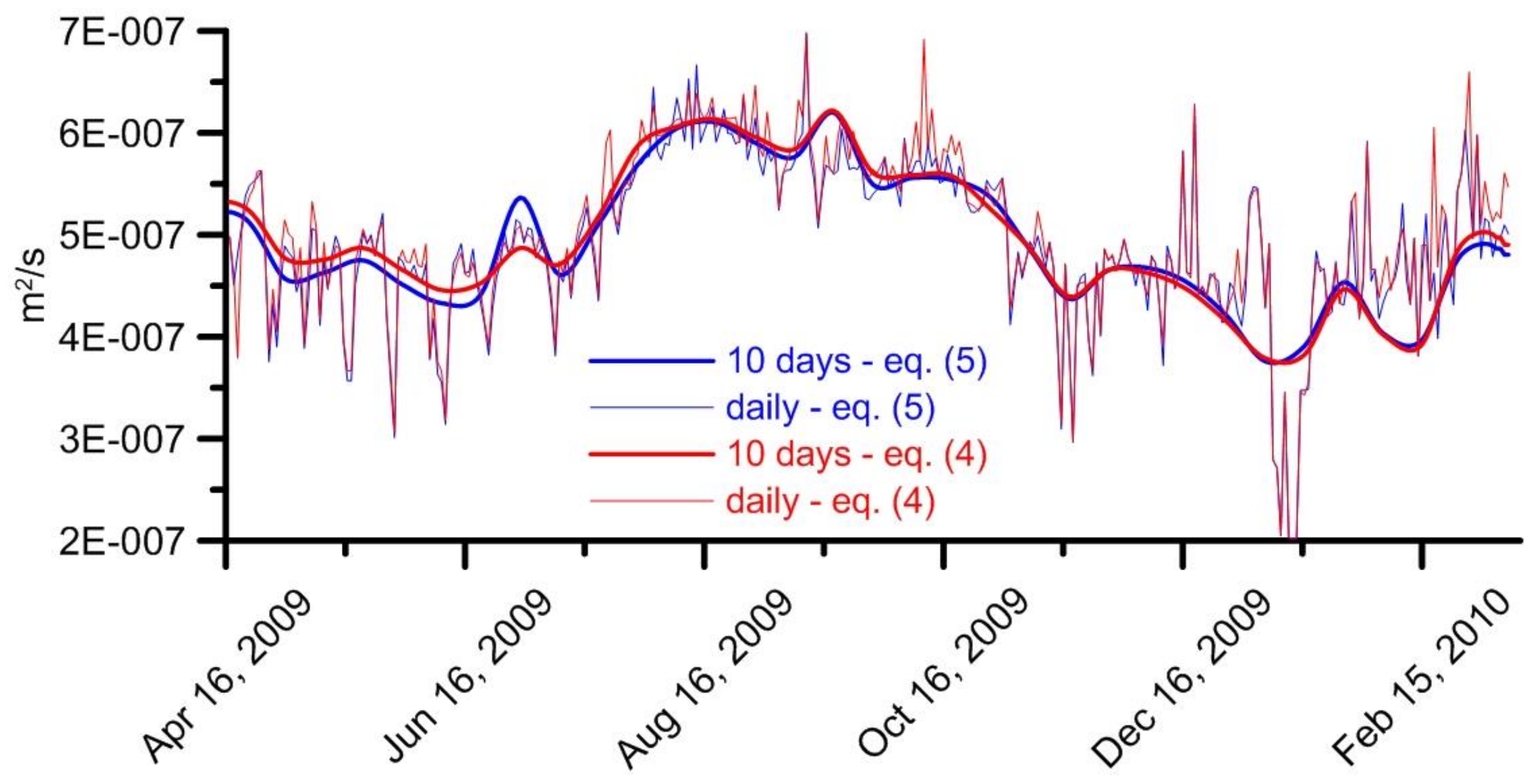

517 Fig. 4 


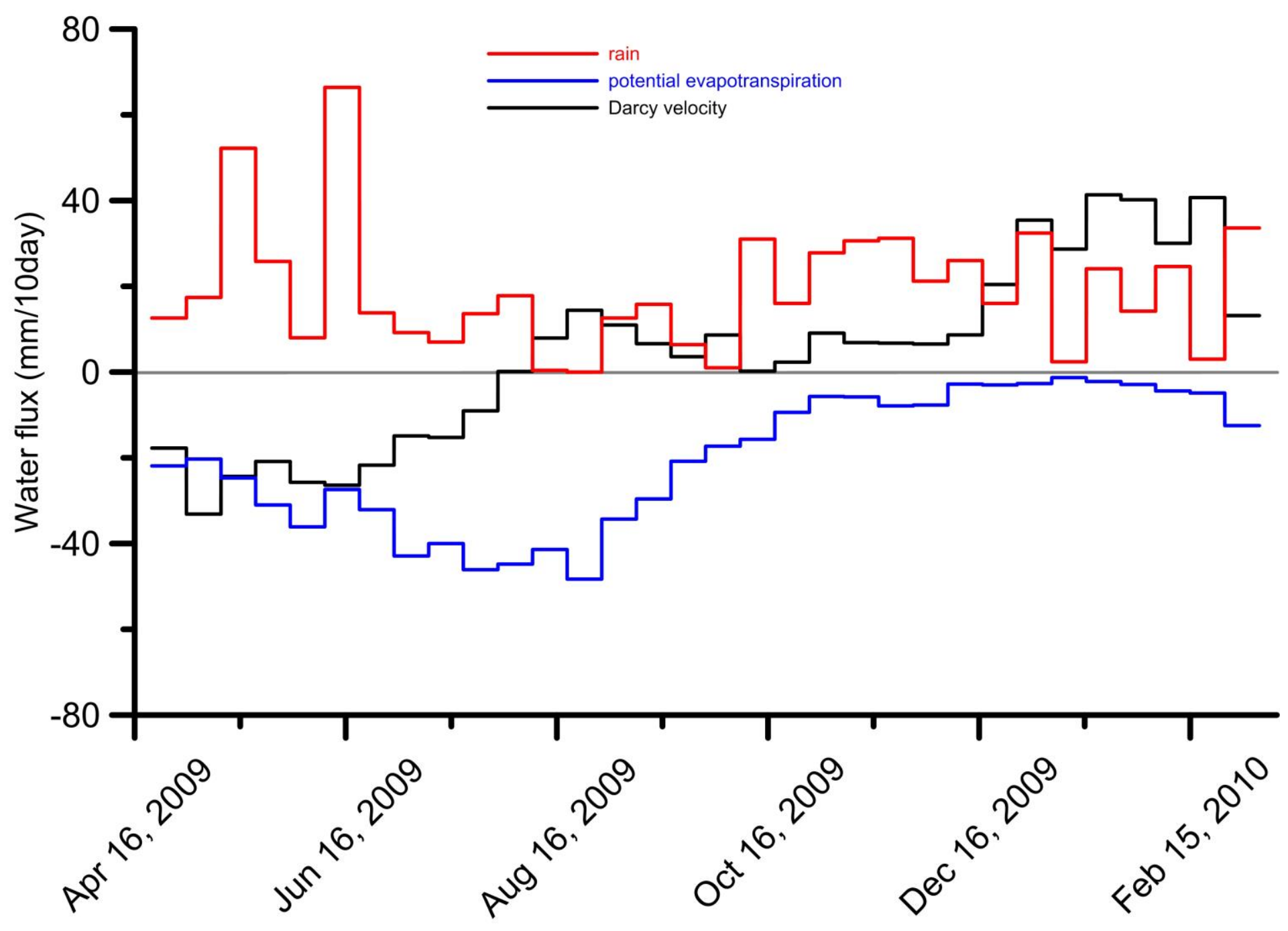

$519 \quad$ Fig. 5 


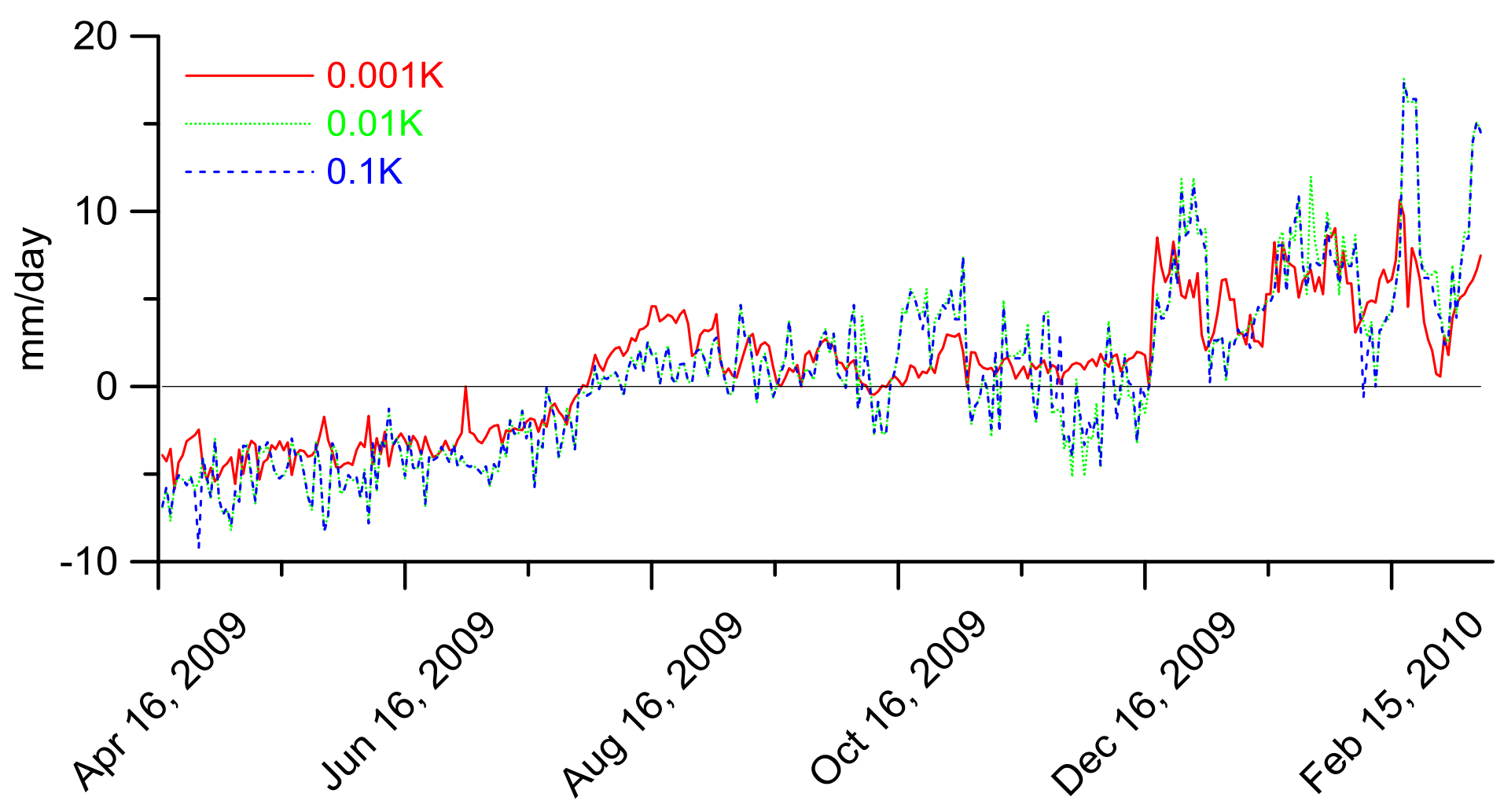

522 Fig. 6a 


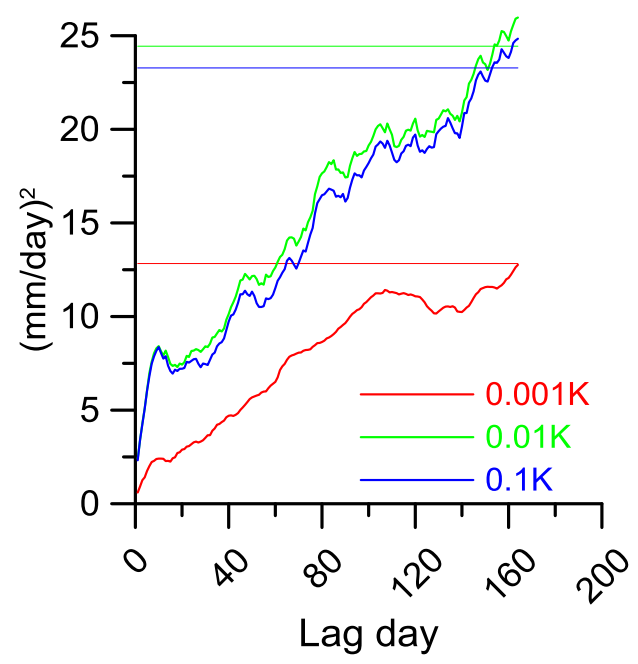

525 Fig. 6b

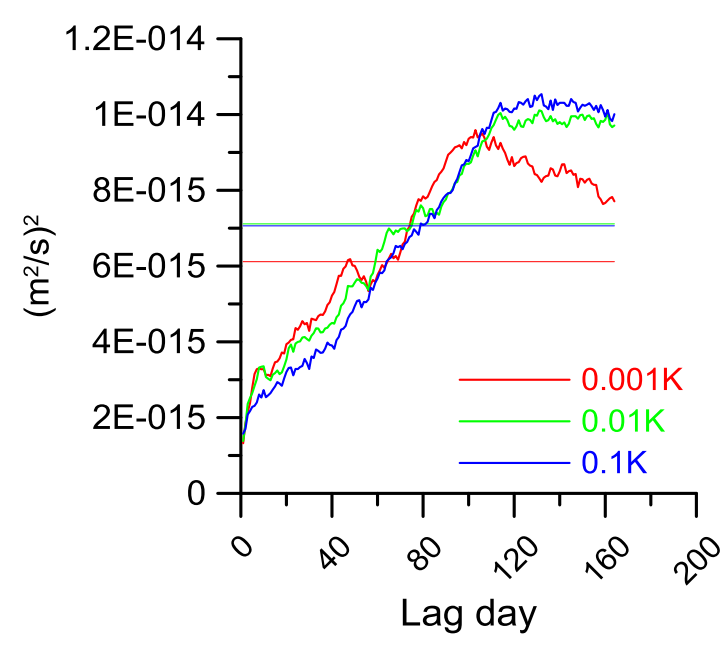

526

Fig. 6c 


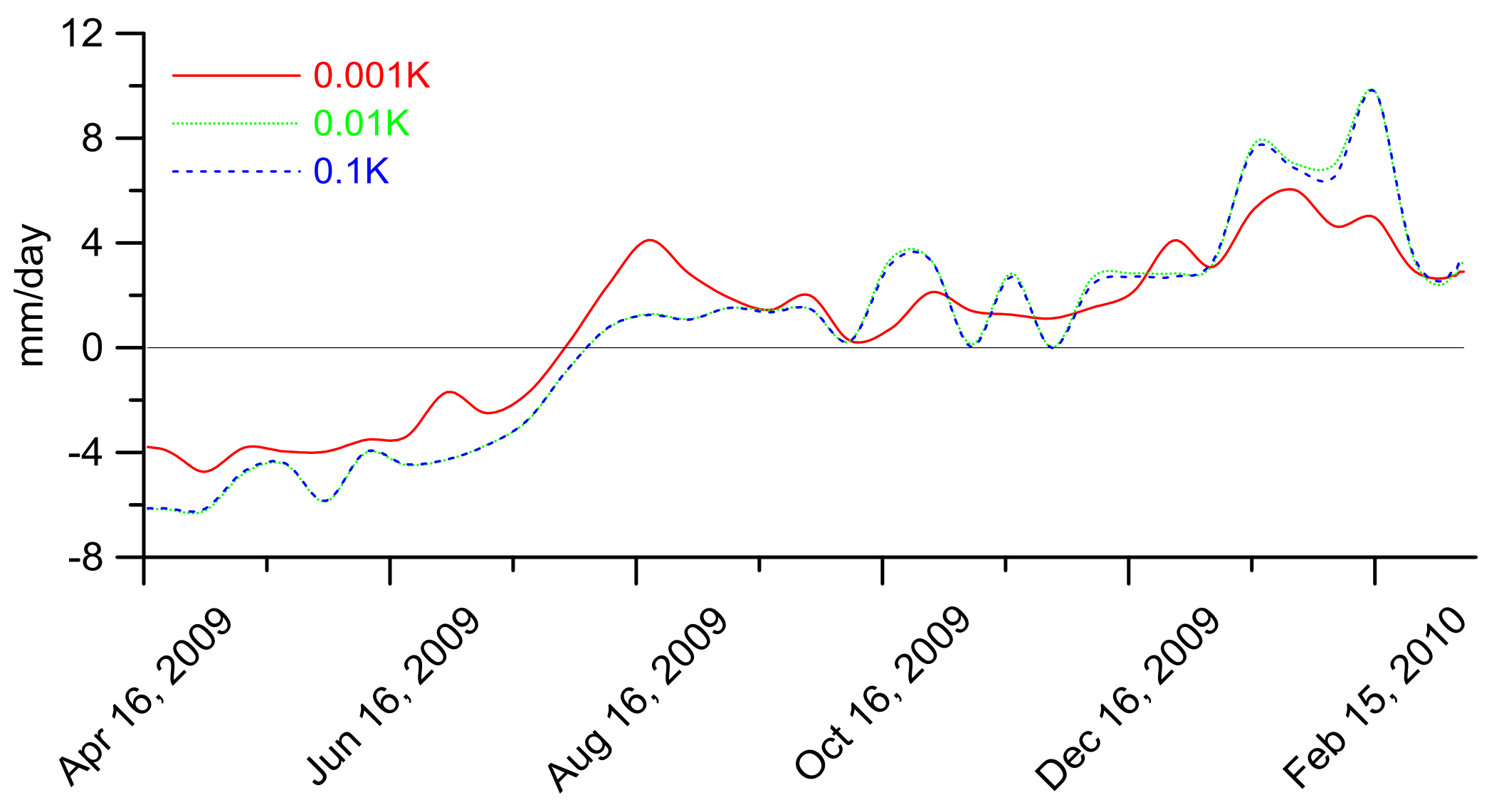

530 Fig. 7a 


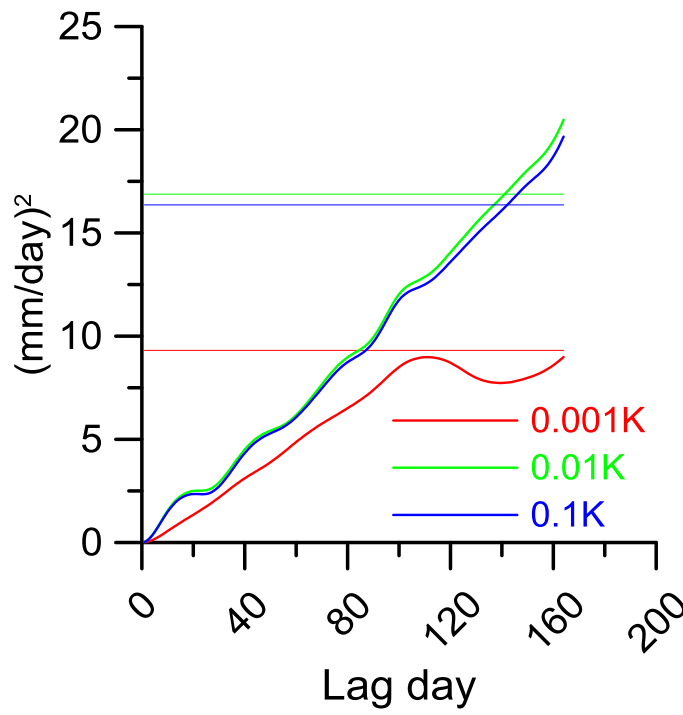

531

Fig. Tb

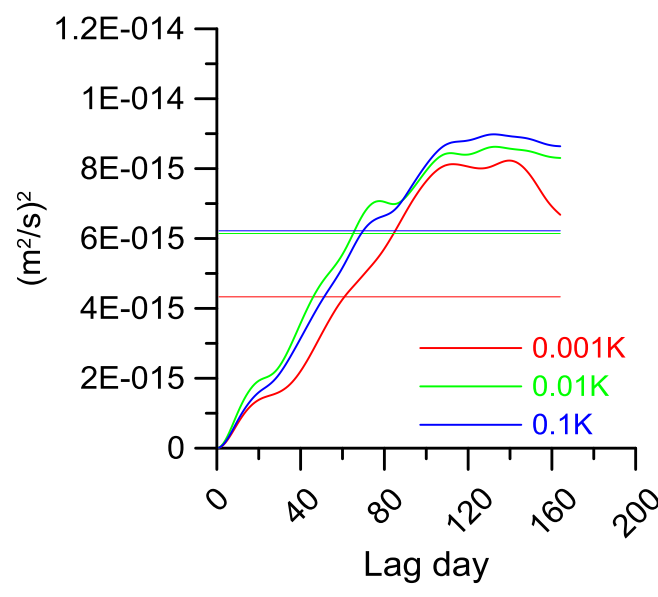

Fig. 7c

533 


\begin{tabular}{|c|c|c|}
\hline \multirow{2}{*}{$\begin{array}{c}\text { Depths of the three sensors } \\
(\mathrm{cm})\end{array}$} & Mean quadratic deviations $\left(\mathrm{mm} \mathrm{d}^{-1}\right)$ \\
& by reference to the calculations achieved with 15,24 and \\
& Eq. (5) & Eq. (4) \\
\hline $14.9,24,34$ & 0.160 & 0.164 \\
\hline $15.1,24,34$ & 0.163 & 0.250 \\
\hline $15,23.9,34$ & 0.289 & 0.350 \\
\hline $15,24.1,34$ & 0.265 & 0.083 \\
\hline $15,24,33.9$ & 0.112 & 0.150 \\
\hline $15,24,34.1$ & 0.146 & \\
\hline
\end{tabular}




\begin{tabular}{|c|c|c|c|c|}
\hline & $\begin{array}{c}\text { Mean } \\
\left(\mathrm{mm} \mathrm{d}^{-1}\right)\end{array}$ & $\begin{array}{c}\text { Standard deviation } \\
\left(\mathrm{mm} \mathrm{d}^{-1}\right)\end{array}$ & $\begin{array}{c}\text { Median } \\
\left(\mathrm{mm} \mathrm{d}^{-1}\right)\end{array}$ & $\begin{array}{c}\text { Interquartile half } \\
\text { distance }\left(\mathrm{mm} \mathrm{d}^{-1}\right)\end{array}$ \\
\hline Eq. (4) 15, & 1.23 & 3.98 & 1.36 & 3.30 \\
\hline $\begin{array}{l}\text { Eq. (5) } 15, \\
24,34 \mathrm{~cm}\end{array}$ & 0.996 & 3.59 & 1.21 & 2.81 \\
\hline
\end{tabular}

540

\begin{tabular}{|c|c|c|c|c|c|}
\hline $\begin{array}{c}\text { Sensors at } \\
15,24,34 \mathrm{~cm}\end{array}$ & $\begin{array}{c}\text { Global } \\
\text { spectrum }\end{array}$ & $\begin{array}{c}\text { First } \\
\text { quarter } \\
\text { from } 0 \text { to } \\
0.125 \mathrm{~d}^{-1}\end{array}$ & $\begin{array}{c}\text { Second } \\
\text { quarter } \\
\text { from } 0.125 \\
\text { to } 0.25 \mathrm{~d}^{-1}\end{array}$ & $\begin{array}{c}\text { Third } \\
\text { quarter } \\
\text { from } 0.25 \\
\text { to } 0.375 \mathrm{~d}^{-1}\end{array}$ & $\begin{array}{c}\text { Fourth } \\
\text { quarter } \\
\text { from } 0.375 \\
\text { to } 0.5 \mathrm{~d}^{-1}\end{array}$ \\
\hline Eq. (4) & 0.954 & 0.960 & 0.109 & 0.048 & 0.142 \\
\hline Eq. (5) & & & & \\
\hline
\end{tabular}

Table 3

542

\begin{tabular}{|c|c|c|c|c|c|}
\hline Eq. (5) & $\begin{array}{c}\text { Global } \\
\text { spectrum }\end{array}$ & $\begin{array}{c}\text { First } \\
\text { quarter } \\
\text { from } 0 \text { to } \\
0.125 \mathrm{~d}^{-1}\end{array}$ & $\begin{array}{c}\text { Second } \\
\text { quarter } \\
\text { from } 0.125 \\
\text { to } 0.25 \mathrm{~d}^{-1}\end{array}$ & $\begin{array}{c}\text { Third } \\
\text { quarter } \\
\text { from } 0.25 \\
\text { to } 0.375 \mathrm{~d}^{-1}\end{array}$ & $\begin{array}{c}\text { Fourth } \\
\text { quarter } \\
\text { from } 0.375 \\
\text { to } 0.5 \mathrm{~d}^{-1}\end{array}$ \\
\hline $15,24,34 \mathrm{~cm}$ & 0.976 & 0.980 & 0.506 & 0.188 & 0.406 \\
and $18,24,32 \mathrm{~cm}$ & & & & \\
\hline
\end{tabular}

Table 4 